\title{
Detection of Intensity and Motion Edges within Optical Flow via Multidimensional Control*
}

\author{
Christoph Brune ${ }^{\dagger}$, Helmut Maurer ${ }^{\dagger}$, and Marcus Wagner ${ }^{\ddagger}$
}

\begin{abstract}
In this paper, we propose a new optimization approach for the simultaneous computation of optical flow and edge detection therein. Instead of using an Ambrosio-Tortorelli type energy functional, we reformulate the optical flow problem as a multidimensional control problem. The optimal control problem is solved by discretization methods and large-scale optimization techniques. The edge detector can be immediately built from the control variables. We provide three series of numerical examples. The first shows that the mere presence of a gradient restriction has a regularizing effect, while the second demonstrates how to balance the regularizing effects of a term within the objective and the control restriction. The third series of numerical results is concerned with the direct evaluation of a TV-regularization term by introduction of control variables with sign restrictions.
\end{abstract}

Key words. optical flow, edge detection, partial differential equation constrained optimization, optimal control problem, direct methods

AMS subject classifications. 35F30, 35R25, 49J20, 49M37, 68U10

DOI. $10.1137 / 080725064$

1. Introduction. A major task in computer vision is the extraction of object and motion information from a given image sequence. In this context, a frequently used concept is that of the optical flow: For consecutive frames, one determines a displacement field (resp., its time derivative), which sets points of equal brightness into correspondence (cf. [4, pp. $250 \mathrm{ff}$.]). The concept of the optical flow finds numerous applications, e.g., for compression of video image data (cf. [21]), automatic retouching of movie sequences during the process of digitalization (cf. [19]), or even reconstruction of three-dimensional surfaces by estimating the disparity map for a stereo image pair ("depth from stereo") (cf. [39]). Its most important applications, however, are connected with object recognition and motion estimation (see, for example, [7], [18], [26], [28], and [40]). Here the discontinuities ("edges") within the optical flow are of particular interest. Ideally, they should be detected without performing an additional step. Moreover, it is reasonable to distinguish within the optical flow between "motion edges," i.e., borders between the projections of moving objects, and "intensity edges," i.e., large gradients of greyscale values within the projections of moving objects.

In the literature, the computation of the optical flow is frequently performed by regularization methods (cf. [37, pp. $53 \mathrm{ff}$.$] ), resulting in the solution of multidimensional variational$

\footnotetext{
* Received by the editors May 23, 2008; accepted for publication (in revised form) June 23, 2009; published electronically November 11, 2009.

http://www.siam.org/journals/siims/2-4/72506.html

${ }^{\dagger}$ Institut für Numerische und Angewandte Mathematik, Westfälische Wilhelms-Universität Münster, Einsteinstr. 62, D-48149 Münster, Germany (christoph.brune@uni-muenster.de, http://www.christoph-brune.de; maurer@ math.uni-muenster.de).

${ }^{\ddagger}$ Corresponding author. Institute for Mathematics and Scientific Computing, University of Graz, Heinrichstrasse 36, A-8010 Graz, Austria (marcus.wagner@uni-graz.at, http://www.thecitytocome.de).
}

1190

Copyright (c) by SIAM. Unauthorized reproduction of this article is prohibited. 
problems. ${ }^{1}$ Then there are two different strategies for a simultaneous edge detection. The first possibility is to replace the objective within the variational problem by a functional of Ambrosio-Tortorelli type, depending on an additional variable $k$ as edge detector. ${ }^{2}$ The second possibility is the addition of convex restrictions for the gradients of the optical flow, thus converting the given variational problem into a multidimensional control problem of the so-called Dieudonné-Rashevsky type. Then the edge detector $k$ can be built immediately from the control variables (cf. [45]).

In the present paper, we pursue the second approach, simultaneously determining the optical flow and the edges therein as a solution of a multidimensional control problem. Our interest in the reformulation and solution of the problem within the framework of optimal control has several reasons. Let us remark first that, from the viewpoint of nonlinear optimization, the present paper (together with [17]) documents for the first time the numerical solution of a control problem of Dieudonné-Rashevsky type by direct methods. ${ }^{3}$ The computations have been carried out within the framework of the diploma thesis [10] of the first author.

The consequences of gradient restrictions have been, to the best of our knowledge, not yet studied within mathematical image processing and are therefore of particular interest. Our numerical experiments demonstrate that the mere presence of a gradient restriction has a pronounced regularizing effect (section 5.1). Next, the control formulation allows us to balance different regularization terms with the control restrictions, so that we may study how the choice of terms and parameters influences the distinction between motion and intensity edges (section 5.2). In a further test series, we solve a control problem with an $L^{1}$-regularization term directly via decomposition of the control variables into positive and negative parts (section 5.3). In order to evaluate our results, we define two new indicators, the "motion edge error" (MEE) and the "intensity edge error" (IEE) (Definitions 4.1 and 4.2). In comparison with our computations, the numerical solution of a variational problem with an AmbrosioTortorelli cost functional gives no significant better results (cf. section 5.4 and [10, pp. $77 \mathrm{ff}$.$] ).$

The outline of this paper is as follows. In section 2, we give the variational formulation and the optimal control reformulation of the optical flow problem. Then in section 3, we provide an existence theorem for control problems of Dieudonné-Rashevsky type, which justifies the application of direct optimization methods. Then we address the discretized problem and its numerical solution by large-scale optimization. The used image data, the visualization, and the evaluation of the results of our computations are described in section 4. Finally, in section 5 we document the results of our numerical experiments.

Notation. Let $\Omega \subset \mathbb{R}^{m}$ be the closure of a strongly Lipschitz domain. Then $C^{k}\left(\Omega, \mathbb{R}^{r}\right)$ denotes the space of $r$-dimensional vector functions $f: \Omega \rightarrow \mathbb{R}^{r}$, whose components are continuous $(k=0)$ or $k$-times continuously differentiable $(k=1, \ldots, \infty)$, respectively; $L^{p}\left(\Omega, \mathbb{R}^{r}\right)$

\footnotetext{
${ }^{1}$ From the extensive literature on this subject, we mention [9], [14], and [47]. The variational formulation of the optical flow problem has already been introduced in [24].

${ }^{2}$ For the image segmentation problem, this approach is well known and has been widely applied. We refer to [1], [2], [5], and [6]. In the context of the optical flow, this method has been less frequently used, e.g., in [33] (however, with quite disappointing numerical results), [41], and [10].

${ }^{3}$ In [13], a transportation flow problem has been numerically solved by methods of combinatorial optimization as the dual problem to a Dieudonné-Rashevsky type problem $(\mathrm{P})_{0}$.
} 
denotes the space of $r$-dimensional vector functions $f: \Omega \rightarrow \mathbb{R}^{r}$, whose components are integrable in the $p$ th power on $\Omega(1 \leqslant p<\infty)$ or are measurable and essentially bounded $(p=\infty)$, respectively; $W_{0}^{1, p}\left(\Omega, \mathbb{R}^{r}\right)$ denotes the Sobolev space of compactly supported $L^{p}\left(\Omega, \mathbb{R}^{r}\right)$ (vector) functions $f: \Omega \rightarrow \mathbb{R}^{r}$, whose components possess first-order weak partial derivatives in $L^{p}(\Omega, \mathbb{R})(1 \leqslant p<\infty)$. Finally, $W_{0}^{1, \infty}\left(\Omega, \mathbb{R}^{r}\right)$ is understood as the Sobolev space of all $r$-vector functions $f: \Omega \rightarrow \mathbb{R}^{r}$ with Lipschitz continuous components and boundary values zero (cf. [15, Theorem 5, p. 131]). Jx denotes the Jacobi matrix of the vector function $x \in W_{0}^{1, p}\left(\Omega, \mathbb{R}^{r}\right)$. The abbreviation " $(\forall) t \in \mathrm{A}$ " has to be read as "for almost all $t \in \mathrm{A}$ " or "for all $t \in \mathrm{A}$ except a Lebesgue null set." The symbol $\mathfrak{o}$ denotes, depending on the context, the zero element of the underlying space.

\section{Variational and optimal control formulation of the optical flow problem.}

2.1. The concept of the optical flow. Let $\Omega \subset \mathbb{R}^{2}$ be a rectangle whose points are denoted by $s=\left(s_{1}, s_{2}\right)^{\mathrm{T}} \in \Omega$. Consider a family of greyscale images $\{I(s, t)\}, 0 \leqslant t \leqslant T$, with identical ranges and zero boundary values, being described through (at least) measurable functions $I: \Omega \times[0, T] \rightarrow \mathbb{R}$ with values $0 \leqslant I(s, t) \leqslant 1(\forall) s \in \Omega, 0 \leqslant t \leqslant T$. Then we search for a phase flux $X(s, t)=\left(X_{1}(s, t), X_{2}(s, t)\right)^{\mathrm{T}}: \Omega \times[0, T] \rightarrow \mathbb{R}^{2}$, which propagates the points of the reference image $I(s, 0)$ under conservation of their brightness, thus obeying the equation

$$
\begin{aligned}
& I\left(s_{1}, s_{2}, t\right)=I\left(s_{1}-X_{1}(s, t), s_{2}-X_{2}(s, t), 0\right), \quad 0 \leqslant t \leqslant T \quad \text { with } \\
& X_{1}(s, 0)=0, \mathrm{X}_{2}(s, 0)=0
\end{aligned}
$$

for all $s=\left(s_{1}, s_{2}\right)^{\mathrm{T}} \in \Omega$. If, moreover, the image data and the vector field $X(s, t)$ depend continuously differentiably on the time variable $t$, then differentiation of (2.1) leads to

$$
I_{t}(s, t)=-I_{s_{1}}(s-X(s, t), 0) \cdot\left(X_{1}\right)_{t}(s, t)-I_{s_{2}}(s-X(s, t), 0) \cdot\left(X_{2}\right)_{t}(s, t),
$$

from which the so-called optical flow constraint (for $t=0$ ) results:

$$
I_{s_{1}}(s, 0)\left(X_{1}\right)_{t}(s, 0)+I_{s_{2}}(s, 0)\left(X_{2}\right)_{t}(s, 0)+I_{t}(s, 0)=0 \quad \forall s \in \Omega .
$$

The vector field $x(s, t)=\left(\left(X_{1}\right)_{t}(s, t),\left(X_{2}\right)_{t}(s, t)\right)^{\mathrm{T}}$ is called optical flow. In practice, the optical flow will be computed for image pairs $I\left(s, t_{N}\right), I\left(s, t_{N+1}\right)$ with $\Delta t=t_{N+1}-t_{N}=1$ as the solution $\left(x_{1}(s), x_{2}(s)\right)^{\mathrm{T}}$ of

$$
I_{s_{1}}\left(s, t_{N}\right) x_{1}(s)+I_{s_{2}}\left(s, t_{N}\right) x_{2}(s)+I_{t}\left(s, t_{N}\right)=0 \quad \forall s \in \Omega
$$

with reference to $t_{N}$. Since neither (2.4) nor (2.5) can determine the optical flow uniquely ("aperture problem"), it will be computed frequently via regularization methods. This means replacing $(2.5)$ by the multidimensional variational problem

$$
\begin{aligned}
(\mathrm{V})_{1}: \quad F\left(x_{1}, x_{2}\right)=\int_{\Omega}( & \left.I_{s_{1}}(s) x_{1}(s)+I_{s_{2}}(s) x_{2}(s)+I_{t}(s)\right)^{2} d s \\
& +\mu \cdot \int_{\Omega} r\left(s, \nabla x_{1}(s), \nabla x_{2}(s)\right) d s \longrightarrow \inf ! ; \quad\left(x_{1}, x_{2}\right) \in W_{0}^{1, p}\left(\Omega, \mathbb{R}^{2}\right)
\end{aligned}
$$

Copyright (C) by SIAM. Unauthorized reproduction of this article is prohibited. 
with $1 \leqslant p<\infty, \mu>0, I \in W_{0}^{1, \infty}\left(\Omega \times\left(t_{N}-\delta, t_{N+1}+\delta\right), \mathbb{R}\right)$, and $r \in C^{2}\left(\Omega \times \mathbb{R}^{4}, \mathbb{R}\right) .^{4}$ Note that the gradient will be computed with respect to the variables $s_{1}$ and $s_{2}$ only ("spatial formulation"), while the dependence of the data and the unknowns on the reference time $t=t_{N}$ has been dropped in notation. The objective consists of a data fidelity term, which minimizes the defect in (2.5), ${ }^{5}$ and a regularization term involving the first partial derivatives of the unknown vector field. In the literature, convex regularization terms have been classified under the categories isotropic/anisotropic (resp., flow driven/image driven), based on the interpretation of the Euler-Lagrange equations for sufficiently smooth solutions of $(\mathrm{V})_{1}$ in terms of diffusion processes (cf. [46]).

2.2. Edge detection within the optical flow. As mentioned in the introduction, simultaneous edge detection within the optical flow may be performed if one replaces the objective in $(\mathrm{V})_{1}$ by a functional of Ambrosio-Tortorelli type. In addition to a (smoothed) version of the optical flow, this functional searches for a function $k$ as a "sketch" for the edges, where $k(s) \approx 0$ or $k(s) \approx 1$, respectively, depending on whether the point $s \in \Omega$ belongs to an edge within $\left(x_{1}, x_{2}\right)$ or not. We arrive at the following rather complicated variational problem:

$$
\begin{aligned}
(\mathrm{V})_{2}: \quad F\left(x_{1}, x_{2}, k\right)= & c_{1}(\varepsilon) \int_{\Omega}\left(I_{s_{1}}(s) x_{1}(s)+I_{s_{2}}(s) x_{2}(s)+I_{t}(s)\right)^{2} d s \\
& +c_{2}(\varepsilon) \int_{\Omega}\left(\left|\nabla x_{1}(s)\right|^{2}+\left|\nabla x_{2}(s)\right|^{2}\right) \cdot\left(k(s)^{2}+c_{4}(\varepsilon)\right) d s \\
& +c_{3}(\varepsilon) \int_{\Omega}\left(\varepsilon \cdot|\nabla k(s)|^{2}+\frac{1}{4 \varepsilon}(1-k(s))^{2}\right) d s \longrightarrow \inf ! ; \\
& \left(x_{1}, x_{2}, k\right) \in W_{0}^{1,2}\left(\Omega, \mathbb{R}^{2}\right) \times W_{0}^{1,2}(\Omega, \mathbb{R})
\end{aligned}
$$

with $I$ as above, $\varepsilon>0$, and weights $c_{i}(\varepsilon)>0,1 \leqslant i \leqslant 4$. The first term within the objective is again a fidelity term connected with (2.5). The second term replaces the classical regularization term of $(\mathrm{V})_{1}$ and realizes a coupling of $x$ and $k$, which favors values $k(s) \approx 0$ in points $s \in \Omega$ with large magnitudes of $\nabla x(s)$. Within the third term, the first member effects a local smoothing of $k$, while the second member enforces $k(s) \approx 1$ except a subset of $\Omega$ of small measure. The interpretation of $k$ as an edge detector is heuristically clear but requires a rigorous justification by proving the $\Gamma$-convergence of the solutions of $(\mathrm{V})_{2}$ towards a solution of a variational problem with an objective of Mumford-Shah type. ${ }^{6}$

As an alternative to the study of $(\mathrm{V})_{2}$, we can reformulate $(\mathrm{V})_{1}$ as an optimal control problem of Dieudonné-Rashevsky type if we add convex restrictions for the gradients $\nabla x_{1}$ and $\nabla x_{2}$ to $(\mathrm{V})_{1}$ :

$(\mathrm{P})_{1}: \quad F(x, u)=\int_{\Omega}\left(I_{s_{1}}(s) x_{1}(s)+I_{s_{2}}(s) x_{2}(s)+I_{t}(s)\right)^{2} d s$

\footnotetext{
${ }^{4}$ In the literature, the problems have been formulated with $x \in B V\left(\Omega, \mathbb{R}^{2}\right)$ as well; see, e.g., [3, pp. 162-174], [22, pp. $81 \mathrm{ff}$.$] , and [27, pp. 9 \mathrm{ff}$.$] .$

${ }^{5}$ Higher order data fidelity terms have been proposed in [8, p. 262 f.] and [34, pp. $\left.143 \mathrm{ff}.\right]$.

${ }^{6}$ In analogy to [5, p. 202, as well as Theorem 2.1, p. 205 f.].
} 
$+\mu \cdot \int_{\Omega} r\left(s, u_{11}(s), u_{12}(s), u_{21}(s), u_{22}(s)\right) d s \longrightarrow \inf ! ; \quad(x, u) \in W_{0}^{1, p}\left(\Omega, \mathbb{R}^{2}\right) \times L^{\infty}\left(\Omega, \mathbb{R}^{4}\right) ;$

$J x(s)=\left(\begin{array}{ll}u_{11}(s) & u_{12}(s) \\ u_{21}(s) & u_{22}(s)\end{array}\right) \quad(\forall) s \in \Omega$

$u \in \mathrm{U}=\left\{\left.u \in L^{p}\left(\Omega, \mathbb{R}^{4}\right)|| u_{11}(s)\right|^{q}+\left|u_{12}(s)\right|^{q}+\left|u_{21}(s)\right|^{q}+\left|u_{22}(s)\right|^{q} \leqslant R^{q}(\forall) s \in \Omega\right\}$.

Here we assume $1 \leqslant p<\infty, 1 \leqslant q<\infty, \mu>0$, and $R>0$. The function $r \in C^{2}\left(\Omega \times \mathbb{R}^{4}, \mathbb{R}\right)$ may be chosen as in $(\mathrm{V})_{1}$. The edge detector $k$ can be immediately constructed from the control variables $u_{i j}$; e.g.,

$$
k(s)=1-\frac{\left|u_{11}(s)\right|^{q}+\left|u_{12}(s)\right|^{q}+\left|u_{21}(s)\right|^{q}+\left|u_{22}(s)\right|^{q}}{\operatorname{Max}_{s \in \Omega}\left(\left|u_{11}(s)\right|^{q}+\left|u_{12}(s)\right|^{q}+\left|u_{21}(s)\right|^{q}+\left|u_{22}(s)\right|^{q}\right)}
$$

(cf. (5.4) below). Consequently, we interpret those subsets of $\Omega$ as "edges" where the control restriction becomes nearly active. Let us remark that the additional gradient restriction can be incorporated consistently into the model of the optical flow, as it excludes only a subset of feasible solutions of (2.5).

\section{Multidimensional control problems of Dieudonné-Rashevsky type.}

3.1. Problem formulation. We consider multidimensional control problems of the shape

$$
(\mathrm{P})_{0}: \quad F(x, u)=\int_{\Omega} f(s, x(s), u(s)) d s \longrightarrow \inf ! ; \quad(x, u) \in W_{0}^{1, p}\left(\Omega, \mathbb{R}^{n}\right) \times L^{p}\left(\Omega, \mathbb{R}^{n m}\right) ;
$$

$$
J x(s)=\left(\begin{array}{ccc}
\frac{\partial x_{1}}{\partial s_{1}}(s) & \ldots & \frac{\partial x_{1}}{\partial s_{m}}(s) \\
\vdots & & \vdots \\
\frac{\partial x_{n}}{\partial s_{1}}(s) & \ldots & \frac{\partial x_{n}}{\partial s_{m}}(s)
\end{array}\right)=\left(\begin{array}{ccc}
u_{11}(s) & \ldots & u_{1 m}(s) \\
\vdots & & \vdots \\
u_{n 1}(s) & \ldots & u_{n m}(s)
\end{array}\right) \quad(\forall) s \in \Omega
$$

$$
u \in \mathrm{U}=\left\{u \in L^{p}\left(\Omega, \mathbb{R}^{n m}\right) \mid u(s) \in \mathrm{K}(\forall) s \in \Omega\right\}
$$

and make the following assumptions about the data of $(\mathrm{P})_{0}$ : Let $n \geqslant 1, m \geqslant 2$, and $1<$ $p<\infty . \Omega \subset \mathbb{R}^{m}$ is the closure of a bounded strongly Lipschitz domain, and the integrand $f(s, \xi, v): \Omega \times \mathbb{R}^{n} \times \mathbb{R}^{n m} \rightarrow \mathbb{R}$ is measurable and essentially bounded with respect to $s$ and continuously differentiable with respect to all $\xi_{i}$ and $v_{i j},{ }^{7}$ while $\mathrm{K} \subset \mathbb{R}^{n m}$ is a convex body with $\mathfrak{o} \in \operatorname{int}(\mathrm{K})$. From these assumptions, we immediately get the existence of a feasible solution (the null function). If $(x, u)$ is admissible in $(\mathrm{P})_{0}$, then $J x(s) \in \mathrm{K}(\forall) s \in \Omega$ implies

\footnotetext{
${ }^{7}$ We will carefully distinguish between the functions $f(s, \xi, v): \Omega \times \mathbb{R}^{n} \times \mathbb{R}^{n m} \rightarrow \mathbb{R}$ and $f(s, x(s), u(s)): \Omega \rightarrow$ $\mathbb{R}$, the latter resulting from the insertion of $x(s)$ and $u(s)$ for the variables $\xi$ and $v$.
}

Copyright (C) by SIAM. Unauthorized reproduction of this article is prohibited. 
$x \in W_{0}^{1, \infty}\left(\Omega, \mathbb{R}^{n}\right) \cap W_{0}^{1, p}\left(\Omega, \mathbb{R}^{n}\right)$. Consequently, in the case $1<p \leqslant m, x$ possesses a Lipschitz representative as well.

\subsection{Existence of global minimizers.}

Theorem 3.1. Consider the problem $(\mathrm{P})_{0}$ under the assumptions of subsection 3.1. If the integrand $f(s, \xi, v): \Omega \times \mathbb{R}^{n} \times \mathbb{R}^{n m} \rightarrow \mathbb{R}$ is convex as a function of $v$ for almost all $s \in \Omega$ and all $\xi \in \mathbb{R}^{n}$, and a growth condition

$$
|f(s, \xi, v)| \leqslant \varphi_{1}(s)+\varphi_{2}(|\xi|,|v|) \quad(\forall) s \in \Omega, \quad \forall(\xi, v) \in \mathbb{R}^{n} \times \mathrm{K}
$$

with $\varphi_{1} \in L^{1}(\Omega, \mathbb{R}), \varphi_{1}(s) \geqslant 0(\forall) s \in \Omega, \varphi_{2} \in C^{0}\left(\mathbb{R}^{n} \times \mathrm{K}, \mathbb{R}\right), \varphi_{2}(|\xi|,|v|) \geqslant 0 \forall(\xi, v) \in$ $\mathbb{R}^{n} \times \mathrm{K}$ is satisfied where $\varphi_{2}$ is a monotonically increasing function in $|\xi|$ as well as in $|v|$, then $(\mathrm{P})_{0}$ admits a global minimizer.

Proof. The proof can be obtained in analogy to [35, pp. 222-224], since, on the one hand, the linear-convex problem $(\mathrm{P})_{0}$ and the relaxed problem $(\overline{\mathrm{P}})_{0}$ studied there are isomorphic, and, on the other hand, by [12, Theorem 8.8, p. 378], the weak* semicontinuity of the objective is still guaranteed if the integrand is a measurable, essentially bounded function of $s$.

The assumptions of Theorem 3.1 are satisfied for all integrands used in the problems in section 5 below; consequently, the optimal control problems admit global minimizers. For instance, due to the assumed Lipschitz continuity of the image data (with constant $C$ ) and the equivalence of the norms in $\mathbb{R}^{r}$, the integrand in (5.5) can be estimated as follows:

$$
\begin{aligned}
\mid( & \left.\left(I_{s_{1}}(s) \xi_{1}+I_{s_{2}}(s) \xi_{2}+I_{t}(s)\right)^{2}+\varepsilon\right)^{1 / 2}+\mu\left(\sum_{i, j=1}^{2}\left|v_{i j}\right|^{p}+\varepsilon\right)^{1 / p} \mid \\
\leqslant & \left(I_{s_{1}}^{2}(s) \xi_{1}^{2}+I_{s_{2}}^{2}(s) \xi_{2}^{2}+I_{t}^{2}(s)\right. \\
& \left.\quad+2\left|I_{s_{1}}(s) \xi_{1} \cdot I_{s_{2}}(s) \xi_{2}+I_{s_{1}}(s) \xi_{1} \cdot I_{t}(s)+I_{s_{2}}(s) \xi_{2} \cdot I_{t}(s)\right|+\varepsilon\right)^{1 / 2} \\
& +\mu\left(\sum_{i, j=1}^{2}\left|v_{i j}\right|^{p}+\varepsilon\right)^{1 / p} \\
\leqslant & \left(C^{2}\left(1+|\xi|^{2}\right)+2 C^{2}\left(\left|\xi_{1}\right|^{2}+\left|\xi_{2}\right|^{2}\right)+2 C^{2}\left(\left|\xi_{1}\right|+\left|\xi_{2}\right|\right)+\varepsilon\right)^{1 / 2} \\
& +\mu\left(\sum_{i, j=1}^{2}\left|v_{i j}\right|^{p}+\varepsilon\right)^{1 / p} \\
\leqslant & \left(3 C^{2}\left(1+|\xi|^{2}\right)+2 C^{2} C_{1}|\xi|^{2}+\varepsilon\right)^{1 / 2}+\mu\left(\sum_{i, j=1}^{2} C_{p}\left|v_{i j}\right|^{2}+\varepsilon\right)^{1 / p} .
\end{aligned}
$$

Consequently, the growth condition (3.4) is satisfied with

$$
\begin{aligned}
& \varphi_{1}(s) \equiv 0 \\
& \varphi_{2}(|\xi|,|v|)=\sqrt{\left(3 C^{2}+2 C^{2} C_{1}\right)\left(1+|\xi|^{2}\right)+\varepsilon}+\mu \sqrt[p]{C_{p}|v|^{2}+\varepsilon}
\end{aligned}
$$

Thus the application of direct methods for the numerical solution of the control problems $(\mathrm{P})_{2}-(\mathrm{P})_{4}$ in section 5 has been justified in principle.

Copyright $\odot$ by SIAM. Unauthorized reproduction of this article is prohibited. 
3.3. Numerical solution of $(\mathbf{P})_{1}$ by direct methods. For the numerical solution of Dieudonné-Rashevsky type problems, we adopt a direct method ("first discretize, then optimize"), thus exploiting, in particular, the simple structure of the state equations (2.9). Following Maurer and Mittelmann [30], [31] and Theißen [42], we convert the multidimensional control problem $(\mathrm{P})_{1}$ into a nonlinear finite-dimensional optimization problem. After an appropriate rescaling, $\Omega$ will be decomposed into $(K \times L)$ pixels with edge length 1 , and the partial derivatives of the image data will be approximated by forward Euler differences. With the abbreviations $I\left(s, t_{N}\right)=I^{(N)}(s), I\left(s, t_{N+1}\right)=I^{(N+1)}(s)$, we get

$$
\begin{aligned}
D_{k l}^{\left(s_{1}\right)}= & \frac{1}{6} \cdot\left(I^{(N)}\left(s_{k+1, l-1}\right)-I^{(N)}\left(s_{k, l-1}\right)+I^{(N)}\left(s_{k+1, l}\right)-I^{(N)}\left(s_{k, l}\right)+I^{(N)}\left(s_{k+1, l+1}\right)\right. \\
& -I^{(N)}\left(s_{k, l+1}\right)+I^{(N+1)}\left(s_{k+1, l-1}\right)-I^{(N+1)}\left(s_{k, l-1}\right)+I^{(N+1)}\left(s_{k+1, l}\right)-I^{(N+1)}\left(s_{k, l}\right) \\
& \left.+I^{(N+1)}\left(s_{k+1, l+1}\right)-I^{(N+1)}\left(s_{k, l+1}\right)\right) \approx I_{s_{1}}\left(s_{k l}\right) ; \\
3.9) & \\
D_{k l}^{\left(s_{2}\right)}= & \frac{1}{6} \cdot\left(I^{(N)}\left(s_{k-1, l+1}\right)-I^{(N)}\left(s_{k-1, l}\right)+I^{(N)}\left(s_{k, l+1}\right)-I^{(N)}\left(s_{k, l}\right)+I^{(N)}\left(s_{k+1, l+1}\right)\right. \\
& -I^{(N)}\left(s_{k+1, l}\right)+I^{(N+1)}\left(s_{k-1, l+1}\right)-I^{(N+1)}\left(s_{k-1, l}\right)+I^{(N+1)}\left(s_{k, l+1}\right)-I^{(N+1)}\left(s_{k, l}\right) \\
& \left.+I^{(N+1)}\left(s_{k+1, l+1}\right)-I^{(N+1)}\left(s_{k+1, l}\right)\right) \approx I_{s_{2}}\left(s_{k l}\right) ; \\
3.10) & \frac{1}{4} \cdot\left(I^{(N+1)}\left(s_{k, l}\right)-I^{(N)}\left(s_{k, l}\right)+I^{(N+1)}\left(s_{k+1, l}\right)-I^{(N)}\left(s_{k+1, l}\right)+I^{(N+1)}\left(s_{k, l+1}\right)\right. \\
D_{k l}^{(t)}= & \left.-I^{(N)}\left(s_{k, l+1}\right)+I^{(N+1)}\left(s_{k+1, l+1}\right)-I^{(N)}\left(s_{k+1, l+1}\right)\right) \approx I_{t}\left(s_{k l}\right) .
\end{aligned}
$$

Defining further

$$
\begin{aligned}
& J_{k l}\left(x_{k l}^{(1)}, x_{k l}^{(2)}, u_{k l}^{(11)}, u_{k l}^{(12)}, u_{k l}^{(21)}, u_{k l}^{(22)}\right) \\
& \quad=\left(D_{k l}^{\left(s_{1}\right)} x_{k l}^{(1)}+D_{k l}^{\left(s_{2}\right)} x_{k l}^{(2)}+D_{k l}^{(t)}\right)^{2}+\mu \cdot r\left(u_{k l}^{(11)}, u_{k l}^{(12)}, u_{k l}^{(21)}, u_{k l}^{(22)}\right),
\end{aligned}
$$

we may state the discretized problem as follows:

$(\mathrm{D})_{1}: \quad \widetilde{F}\left(x_{11}^{(1)}, \ldots, x_{K L}^{(1)}, x_{11}^{(2)}, \ldots, x_{K L}^{(2)}, u_{11}^{(11)}, \ldots, u_{K L}^{(11)}, u_{11}^{(12)}, \ldots, u_{K L}^{(12)}, u_{11}^{(21)}, \ldots, u_{K L}^{(21)}\right.$,

$$
=\frac{1}{4} \sum_{k=3}^{K-2} \sum_{l=3}^{L-2}\left(J_{k-1, l}(\ldots)+J_{k, l}(\ldots)+J_{k-1, l-1}(\ldots)+J_{k, l-1}(\ldots)\right) \longrightarrow \inf ! ;
$$

$\left(x_{11}^{(1)}, \ldots, u_{K L}^{(22)}\right) \in \mathbb{R}^{6 K L}$;

$$
\left(\begin{array}{ll}
u_{k l}^{(11)} & u_{k l}^{(12)} \\
u_{k l}^{(21)} & u_{k l}^{(22)}
\end{array}\right)=\left(\begin{array}{ll}
x_{k+1, l}^{(1)}-x_{k, l}^{(1)} & x_{k, l+1}^{(1)}-x_{k, l}^{(1)} \\
x_{k+1, l}^{(2)}-x_{k, l}^{(2)} & x_{k, l+1}^{(2)}-x_{k, l}^{(2)}
\end{array}\right) \quad \forall k, l
$$

$\left|u_{k l}^{(11)}\right|^{q}+\left|u_{k l}^{(12)}\right|^{q}+\left|u_{k l}^{(21)}\right|^{q}+\left|u_{k l}^{(22)}\right|^{q} \leqslant R^{q} \quad \forall k, l$. 
The necessary optimality conditions (Karush-Kuhn-Tucker conditions) for (D) 1 (cf. [23, p. $305 \mathrm{f}$.$] ) result in the evaluation of large systems of nonlinear equations, which can be solved$ with high precision and efficiency by interior-point methods (see, for example, [25]). In detail, we used MATLAB as the input/output platform. The discretized problem has been formulated with the aid of the modelling language $\mathrm{AMPL}^{8}$ and then transferred to the interior-point solver IPOPT (cf. [29] and [44]). The results have been represented, evaluated, and archived with MATLAB again. For the convergence analysis of our method, we refer the reader to [17].

\section{Visualization and evaluation of the results.}

4.1. Documentation of the image data. For our numerical experiments, three image sequences have been chosen: the Rotating Sphere Sequence, ${ }^{9}$ the New Marbled Block Sequence, and the Hamburg Taxi Sequence. ${ }^{10}$ The Rotating Sphere Sequence has been generated artificially and shows a textured ball, which rotates by its own axis against a background, which shows some texture as well. For this sequence, ground-truth data are available, which can be used for the evaluation of the quality of edge detection. Our computations are related to the passage of frame 13 to frame 14 (see Figures 1-4).

In the New Marbled Block Sequence, which has been artificially generated as well, two marble boulders move in different directions while a third one rests unmoved. Since the scenery is illuminated by several light sources, the boulders cast multiple interfering shadows. Ground-truth is available for this sequence as well. Our computations are related to the passage of frame 163 to frame 164. In order to deal with a comparable number of pixels, we restrict ourselves to an appropriate segment (see Figures 5-6).

The advantages of these artificial sequences consist in the simple structure of objects and motions as well as in the absence of background noise. Thus both sequences are excellent candidates for a comparative analysis of methods with simultaneous edge detection. Our third sequence is the well-known Hamburg Taxi Sequence, which stems from a real scene but is afflicted, for this reason, with considerable noise. We relate our computations to the passage of frame 22 to frame 23 (see Figures 7-8).

4.2. Visualization of the results. The visualization of the optical flow $\left(x_{1}(s), x_{2}(s)\right)^{\mathrm{T}}$ as a vector field (see Figure 3) by plotting the arrows often appears unsatisfactory. A more instructive representation will be given by a so-called colorful orientation plot where the direction of the flow is coded by the color of a pixel, while its intensity increases with the magnitude of the flow vector. The correspondence between color and orientation can be read from the colored border as a legend (see Figure 4). In the present paper, this visualization has been realized within an HSI color model (cf. [36, pp. $25 \mathrm{ff}$.$] ), where every color is represented$ by the three coordinates hue, saturation, and intensity. Since for the visualization of the optical flow only two coordinates are needed, we took a constant saturation. In section 5, we

\footnotetext{
${ }^{8} \mathrm{AMPL}$ is a commercially distributed modelling language, which allows for the description of large-scale optimization problems, the interaction with a solver, and the further processing of the output (cf. [16]).

${ }^{9}$ The sequence is documented in [32] and can be accessed via http://of-eval.sourceforge.net (last access: 27.02.2008)

${ }^{10}$ The sequences are accessible via http://i21www.ira.uka.de/image_sequences/download_tar.cgi?marmor_ stat (last access: 27.02.2008) and http://i21www.ira.uka.de/image_sequences/download_zip.cgi?taxi (last access: 27.02.2008), respectively. For details, please contact nagel@iaks.uni-karlsruhe.de.
}

Copyright (C) by SIAM. Unauthorized reproduction of this article is prohibited. 


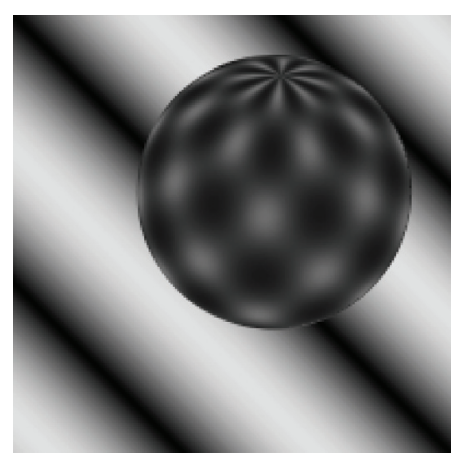

Figure 1.

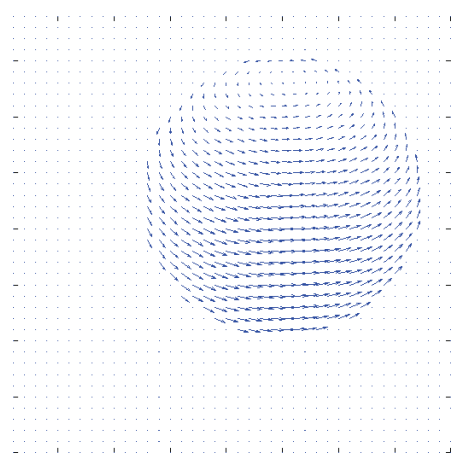

Figure 3.

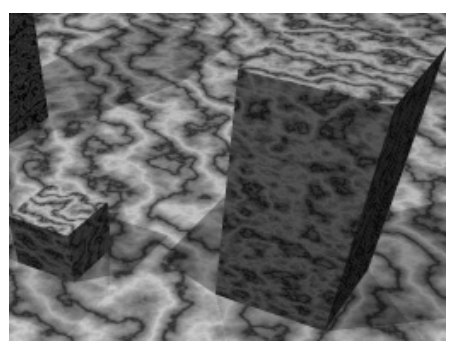

Figure 5.

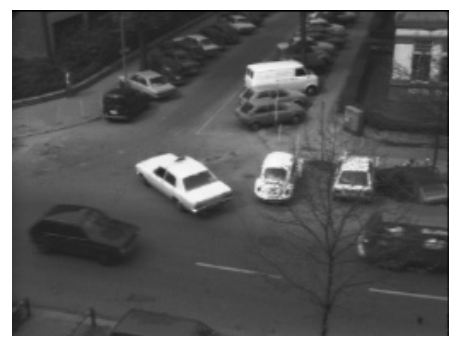

Figure 7.

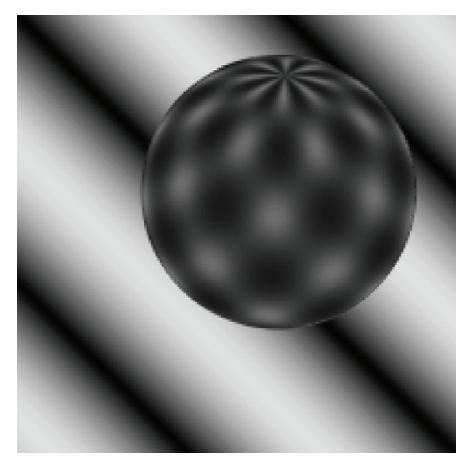

Figure 2.

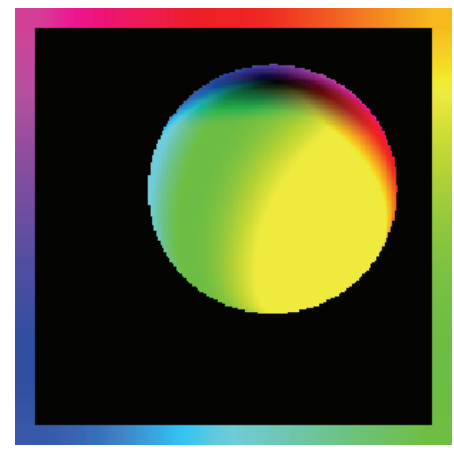

Figure 4.

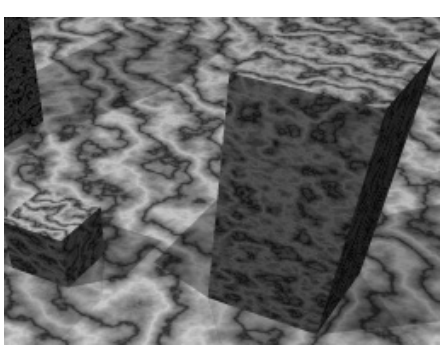

Figure 6.

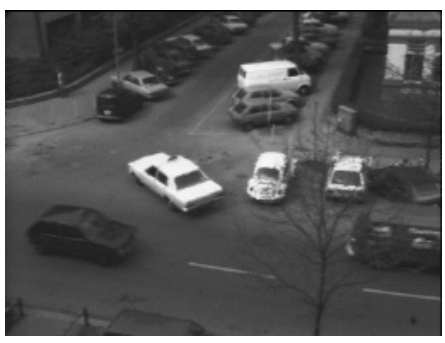

Hamburg Taxi Sequence: frames 22 (left) and 23 (right).

Rotating Sphere Sequence: frames 13 (left) and 14 (right).

Rotating Sphere Sequence: vector plot (left) and colorful orientation plot (right) of the ground-truth data $\hat{x}$ for the passage of frame 13 to frame 14.

New Marbled Block Sequence: Segments of frames 163 (left) and 164 (right).

Figure 8 . 
will illustrate our results with a colorful orientation plot for the optical flow $x(s)$ as well as with a greyscale image of the edge detector $k(s)$.

4.3. Criteria for evaluation of our results. The computation of the optical flow will be evaluated by means of the "average angular error" (AAE), which will be determined from the ground-truth vector field $\hat{x}(s)$ and the calculated vector field $x(s)$ by the formula

$$
\begin{array}{rl}
A & A E(\hat{x}, x) \\
& =\frac{1}{K L} \sum_{k=1}^{K} \sum_{l=1}^{L} \arccos \left(\frac{\hat{x}_{1}\left(s_{k l}\right) x_{1}\left(s_{k l}\right)+\hat{x}_{2}\left(s_{k l}\right) x_{2}\left(s_{k l}\right)+1}{\sqrt{\left(\hat{x}_{1}\left(s_{k l}\right)\right)^{2}+\left(\hat{x}_{2}\left(s_{k l}\right)\right)^{2}+1} \sqrt{\left(x_{1}\left(s_{k l}\right)\right)^{2}+\left(x_{2}\left(s_{k l}\right)\right)^{2}+1}}\right) \\
& \approx \frac{1}{|\Omega|} \int_{\Omega} \arccos \left(\frac{\hat{x}_{1}(s) x_{1}(s)+\hat{x}_{2}(s) x_{2}(s)+1}{\sqrt{\left(\hat{x}_{1}(s)\right)^{2}+\left(\hat{x}_{2}(s)\right)^{2}+1} \sqrt{\left(x_{1}(s)\right)^{2}+\left(x_{2}(s)\right)^{2}+1}}\right) d s
\end{array}
$$

for a discretization with $(K \times L)$ pixels. The AAE criterion has been widely accepted now (cf., e.g., [32, p. 135] and [48, p. 251]) and will be used here in order to ensure comparability.

Criteria for the evaluation of edge sketches $k$, however, are missing in the literature as yet. For this reason, we propose two new evaluation criteria, distinguishing between motion and intensity edges. In order to rate the quality of motion edge detection, we rely on the ground-truth data $\hat{x}$ of the image sequence and represent $|\hat{x}|$ as greyscale image $G$ ("flow field magnitude representation"). Applying a static edge detection to $G$, we get an edge sketch $k_{m}$ with values in the interval $[0,1]$. Now we define the following error measure MEE.

Definition 4.1 (motion edge error (MEE)). Assume that an edge sketch $k_{m}: \Omega \rightarrow[0,1]$ for the normed magnitude $|\hat{x}|$ of the ground-truth data and an edge sketch $k: \Omega \rightarrow[0,1]$ for the computed optical flow $x$ are given. Then we define

$$
\operatorname{MEE}(\hat{x}, x)=\sum_{k=1}^{K} \sum_{l=1}^{L}\left(k_{m}\left(s_{k l}\right)-k\left(s_{k l}\right)\right)^{2} \approx \int_{\Omega}\left(k_{m}(s)-k(s)\right)^{2} d s
$$

for a discretization with $(K \times L)$ pixels.

The quality of intensity edge detection can be evaluated after an immediate static edge detection within the frames $I\left(s, t_{N}\right)$ and $I\left(s, t_{N+1}\right)$, resulting in edge sketches $k_{g}^{(N)}, k_{g}^{(N+1)}$ with values in $[0,1]$. With the aid of both of them, we define a further error measure IEE.

Definition 4.2 (intensity edge error (IEE)). Assume that edge sketches $k_{g}^{(N)}, k_{g}^{(N+1)}: \Omega \rightarrow$ $[0,1]$ for the greyscale images $I\left(s, t_{N}\right), I\left(s, t_{N+1}\right)$, and an edge sketch $k: \Omega \rightarrow[0,1]$ for the computed optical flow $x$ are given. Then we define

$$
\begin{aligned}
\operatorname{IEE}(\hat{x}, x) & =\sum_{k=1}^{K} \sum_{l=1}^{L} \frac{1}{2}\left(\left(k_{g}^{(N)}\left(s_{k l}\right)-k\left(s_{k l}\right)\right)^{2}+\left(k_{g}^{(N+1)}\left(s_{k l}\right)-k\left(s_{k l}\right)\right)^{2}\right) \\
& \approx \int_{\Omega} \frac{1}{2}\left(\left(k_{g}^{(N)}(s)-k(s)\right)^{2}+\left(k_{g}^{(N+1)}(s)-k(s)\right)^{2}\right) d s
\end{aligned}
$$

for a discretization with $(K \times L)$ pixels.

Copyright $\odot$ by SIAM. Unauthorized reproduction of this article is prohibited. 


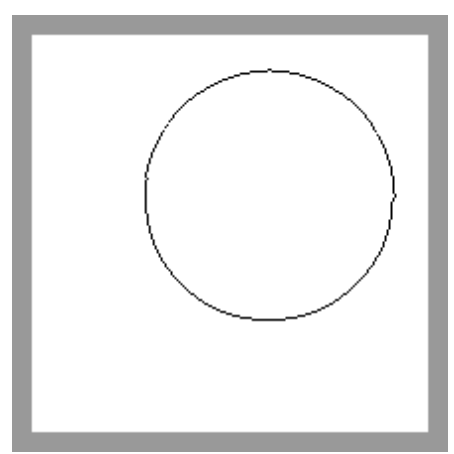

Figure 9.

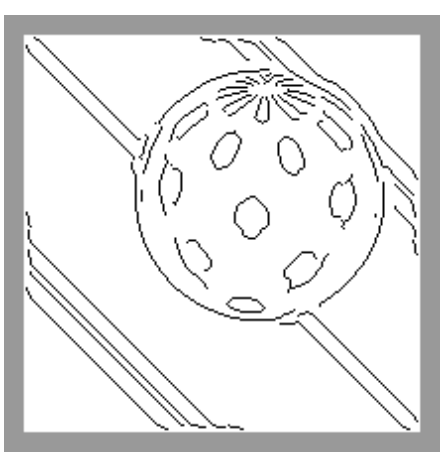

Figure 10.
Rotating Sphere Sequence: Edge sketches $k_{m}$ (left) and $k_{g}^{(N)}$ (right).

These new criteria allow us to distinguish whether a given method enhances motion edges or the textures within the objects. In Figures 9 and 10, the edge sketches $k_{m}$ and $k_{g}^{(N)}$ for the passage within the Rotating Sphere Sequence are depicted.

Here $k_{m}$ has been computed with a Sobel operator. In order to find $k_{g}^{(N)}$ and $k_{g}^{(N+1)}$, a Canny detector with a threshold 0.085 and a standard deviation of 0.1 for the underlying Gaussian filter has been used.

\section{Numerical results.}

5.1. First series: Objective without regularization term. In our first series of numerical experiments, we consider an objective without regularization term, in order to study the immediate influence of the control restriction. $\mathrm{K}$ is the norm body of the $q$-Hölder norm in the space $\mathbb{R}^{4}$. The resulting control problem reads as follows:

$$
\begin{gathered}
(\mathrm{P})_{2}: \quad F(x, u)=\int_{\Omega}\left(I_{s_{1}}(s) x_{1}(s)+I_{s_{2}}(s) x_{2}(s)+I_{t}(s)\right)^{2} d s \longrightarrow \inf ! ; \\
(x, u) \in W_{0}^{1, \infty}\left(\Omega, \mathbb{R}^{2}\right) \times L^{\infty}\left(\Omega, \mathbb{R}^{4}\right) ; \\
J x(s)=\left(\begin{array}{ll}
u_{11}(s) & u_{12}(s) \\
u_{21}(s) & u_{22}(s)
\end{array}\right) \quad(\forall) s \in \Omega ; \\
\left|u_{11}(s)\right|^{q}+\left|u_{12}(s)\right|^{q}+\left|u_{21}(s)\right|^{q}+\left|u_{22}(s)\right|^{q} \leqslant R^{q} \quad(\forall) s \in \Omega .
\end{gathered}
$$

The edge detector is defined as

$$
k(s)=1-\frac{\left|u_{11}(s)\right|^{q}+\left|u_{12}(s)\right|^{q}+\left|u_{21}(s)\right|^{q}+\left|u_{22}(s)\right|^{q}}{\operatorname{Max}_{s \in \Omega}\left(\left|u_{11}(s)\right|^{q}+\left|u_{12}(s)\right|^{q}+\left|u_{21}(s)\right|^{q}+\left|u_{22}(s)\right|^{q}\right)} .
$$

During our tests, the parameters $q$ and $R$ (type and sharpness of the norm body) have been varied (see Figures 11-16). We may summarize that the presence of a control restriction for $u=J x$ is in a sense comparable with a classical regularization. On the one hand, Figures 1116 clearly show the smoothing effect of the restriction, while, on the other hand, the quality of the edge detection depends very sensitively on the choice of $R$. A sharp restriction results in a strong, uniform regularization of the optical flow and a bold edge sketch even with some

Copyright (C) by SIAM. Unauthorized reproduction of this article is prohibited. 
tendency to oversmoothing. Choosing, in contrary, a large norm body, e.g., $R=4$ (Figure 11), we get a weaker regularization effect and a more precise, but as well much more faded, edge sketch. If one is interested mainly in the detection of intensity edges, i.e., in the texture of moving objects, then this approach could be pursued. For different kinds of norm bodies, i.e., under variation of $q$, an analogous behavior has been observed (cf. [10, pp. $104 \mathrm{f}$. and 124]). Note that the maximum function in (5.4) is available within the AMPL implementation.

5.2. Second series: Objective with $L^{p}$-regularization term, $p>1$. In our second test series, we focus on the detection of motion edges within the optical flow. Consequently, we choose an objective with a robust data term together with an isotropic, flow-driven regularization term. ${ }^{11} \mathrm{~K}$ is again the norm body of the $q$-norm in $\mathbb{R}^{4}$. The investigated control problem reads as follows:

$$
\begin{array}{ll}
(\mathrm{P})_{3}: \quad & F(x, u)=\int_{\Omega}\left(\left(I_{s_{1}}(s) x_{1}(s)+I_{s_{2}}(s) x_{2}(s)+I_{t}(s)\right)^{2}+\varepsilon\right)^{1 / 2} d s \\
+ & \mu \cdot \int_{\Omega}\left(\left|u_{11}(s)\right|^{p}+\left|u_{12}(s)\right|^{p}+\left|u_{21}(s)\right|^{p}+\left|u_{22}(s)\right|^{p}+\varepsilon\right)^{1 / p} d s \longrightarrow \inf ! \\
& (x, u) \in W_{0}^{1, \infty}\left(\Omega, \mathbb{R}^{2}\right) \times L^{\infty}\left(\Omega, \mathbb{R}^{4}\right) ;
\end{array}
$$

$$
\begin{aligned}
& J x(s)=\left(\begin{array}{ll}
u_{11}(s) & u_{12}(s) \\
u_{21}(s) & u_{22}(s)
\end{array}\right) \quad(\forall) s \in \Omega \\
& \left|u_{11}(s)\right|^{q}+\left|u_{12}(s)\right|^{q}+\left|u_{21}(s)\right|^{q}+\left|u_{22}(s)\right|^{q} \leqslant R^{q} \quad(\forall) s \in \Omega
\end{aligned}
$$

while the edge detector

$$
k(s)=\frac{\kappa(s)}{\operatorname{Max}_{s \in \Omega} \kappa(s)} \cdot\left\{\begin{array}{l|l}
1 & \kappa(s) \geqslant \alpha R^{q} \\
0 & \kappa(s)<\alpha R^{q}
\end{array} ; \quad \text { with } \kappa(s)=\left|u_{11}(s)\right|^{q}+\left|u_{12}(s)\right|^{q}+\left|u_{21}(s)\right|^{q}+\left|u_{22}(s)\right|^{q}\right.
$$

has been used. Within our multidimensional control problem, there are five parameters. For the computations with the practically noiseless data of the Rotating Sphere Sequence, the choice of the robustness parameter $\varepsilon>0$ is of comparably minor importance. $p>1$ and $\mu>0$ define the kind and strength of the regularization; $q$ and $R$ describe as in the subsection above the geometry of the norm body restricting the gradient of the optical flow. The definition of the edge detector comprises a further parameter $\alpha$, which may be understood as a threshold. From the plenty of results documented in [10], we present a sequence of computations where only $R$ varies, while $\varepsilon, \mu, p$ and $q$ are fixed (Figures 17-24). For the last computation, we show three edge sketches generated with different values of $\alpha$ (Figures 23-28). The smoothing effects of the regularization term and the control restriction are present at the same time; for large values of $R$ (in this example for $R>2$ ), the influence of the control restriction decreases. When experimenting with "adjoint" pairs of parameters $p \neq 2, p^{-1}+q^{-1}=1$, a noticeable

\footnotetext{
${ }^{11}$ Cf. [8, p. 261 f.], [9, p. 217], and [10, p. 94].
} 
improvement of convergence and quality of the results could not be observed (cf. [10, pp. 108 and 124]).

5.3. Third series: Objective with $L^{1}$-regularization term. The optimal control reformulation of the optical flow problem allows for treating problems with $L^{1}$ - (resp., TV-) regularization terms ${ }^{12}$ directly by decomposition of the control variables into positive and negative parts: $:^{13}$

$$
\begin{aligned}
& u_{i j}(s)=u_{i j}^{+}(s)-u_{i j}^{-}(s) \quad \text { with } \quad u_{i j}^{+}(s) \geqslant 0, u_{i j}^{-}(s) \geqslant 0 \quad(\forall) s \in \Omega \quad \Longrightarrow \\
& \left|u_{i j}(s)\right|=u_{i j}^{+}(s)+u_{i j}^{-}(s) \quad(\forall) s \in \Omega .
\end{aligned}
$$

Thus we arrive at the following control problem with a 1-norm body:

$$
\begin{aligned}
& (\mathrm{P})_{4}: \quad F(x, u)=\int_{\Omega}\left(\left(I_{s_{1}}(s) x_{1}(s)+I_{s_{2}}(s) x_{2}(s)+I_{t}(s)\right)^{2}+\varepsilon\right)^{1 / 2} d s \\
& +\mu \cdot \int_{\Omega} \sum_{i, j=1}^{2}\left(u_{i j}^{+}(s)+u_{i j}^{-}(s)\right) d s \longrightarrow \inf ! ; \quad(x, u) \in W_{0}^{1, \infty}\left(\Omega, \mathbb{R}^{2}\right) \times L^{\infty}\left(\Omega, \mathbb{R}^{8}\right) \\
& J x(s)=\left(\begin{array}{ll}
u_{11}^{+}(s)-u_{11}^{-}(s) & u_{12}^{+}(s)-u_{12}^{-}(s) \\
u_{21}^{+}(s)-u_{21}^{-}(s) & u_{22}^{+}(s)-u_{22}^{-}(s)
\end{array}\right) \quad(\forall) s \in \Omega ; \\
& \sum_{i, j=1}^{2}\left(u_{i j}^{+}(s)+u_{i j}^{-}(s)\right) \leqslant R \quad(\forall) s \in \Omega ; \\
& u_{i j}^{+}(s), u_{i j}^{-}(s) \geqslant 0 \quad(\forall) s \in \Omega, \quad 1 \leqslant i, j \leqslant 2 .
\end{aligned}
$$

The objective again involves a robust data term. As the edge detector, we define

$$
k(s)=1-\frac{\sum_{i, j=1}^{2}\left(u_{i j}^{+}(s)+u_{i j}^{-}(s)\right)}{\operatorname{Max}_{s \in \Omega} \sum_{i, j=1}^{2}\left(u_{i j}^{+}(s)+u_{i j}^{-}(s)\right)} .
$$

In Figures 29-34, selected results are depicted. In consequence of the $L^{1}$ - (resp., TV-) regularization, we obtain an excellent, in comparison with the second test series even sharper, view of the motion edges (compare, e.g., Figure 22 with Figure 30). At the same time, the typical staircasing effect appears.

5.4. Comparison with results from the Ambrosio-Tortorelli method. In order to compare our optimal control approach with a state-of-art variational method, the variational problem $(\mathrm{V})_{2}$ with Ambrosio-Tortorelli objective has been solved numerically as well (cf. [10, pp. $75 \mathrm{ff}$.] for the details of the implementation). We present the examples with the best MEE

\footnotetext{
${ }^{12}$ In the literature, problems of this type have been numerically solved either by passage to the dual problem or by replacing the $L^{1}$-regularization term by $\int_{\Omega} \sqrt{\left|\nabla x_{1}(t)\right|^{2}+\left|\nabla x_{2}(t)\right|^{2}+\varepsilon} d t$; cf. [11], [20], and the papers cited in footnote 4 above.

${ }^{13}$ Cf. [38, p. 121 f.] as well as [43, p. 307, (16)], where the same decomposition has been applied for the numerical solution of one-dimensional control problems with an objective involving an $L^{1}$-norm term.
}

Copyright $\odot$ by SIAM. Unauthorized reproduction of this article is prohibited. 
and IEE values achieved for the Rotating Sphere Sequence (Figures 35 and 36) and the New Marbled Block Sequence (Figures 37 and 38). A quantitative comparison shows that the error measures produced by the optimal control method have a comparable range (cf. Figures 20, 30, and 36) or are even slightly better (compare Figure 32 with Figure 38) than the values given by the Ambrosio-Tortorelli method. A drawback shared by both methods is the necessity for the empirical determination of the parameters within the objectives. In the optimal control approach, however, we must deal essentially with $\mu$ and $R$ (in the second series, the robustness parameter $\varepsilon$ as well as $p, q$, and $\alpha$ may be fixed in advance), while the Ambrosio-Tortorelli method involves the simultaneous adaptation of five parameters. With respect to the computing time used, the optimal control method turned out to be the slower one, due at least partially to the modularized implementation. In the framework of the present investigations, however, we made no rigorous attempt at tuning.

5.5. Conclusion. Our experiments demonstrate that the calculation of the optical flow with simultaneous edge detection in the framework of multidimensional optimal control offers a real alternative to the Ambrosio-Tortorelli approach of the calculus of variations. Even though the addition of a mere control restriction provides a regularizing effect, it proved to be convenient to incorporate a regularizing term into the objective at the same time. As a further desirable feature of the optimal control approach, the edge detector $k$ can be easily adapted or modified via additional parameters. Finally, the optimal control formulation allows a primal evaluation of TV-regularization terms by introduction of control variables with sign restrictions. Further investigation should comprise experiments with anisotropic norm bodies as well as the incorporation of a priori available information (e.g., about direction and velocity of the motion of observed objects) as additional state and control restrictions.

Copyright $\odot$ by SIAM. Unauthorized reproduction of this article is prohibited. 
5.6. Illustrations.

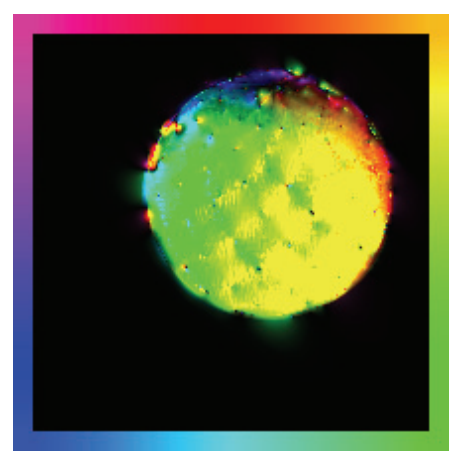

Figure 11.

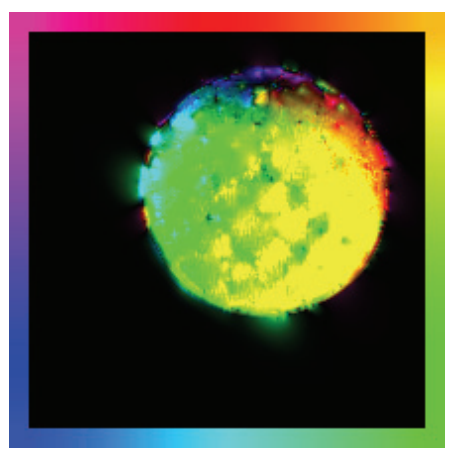

Figure 13.

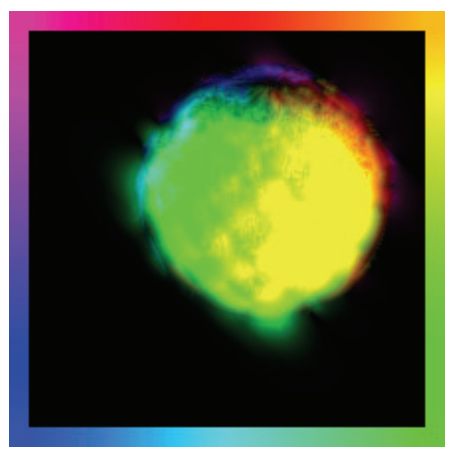

Figure 15.

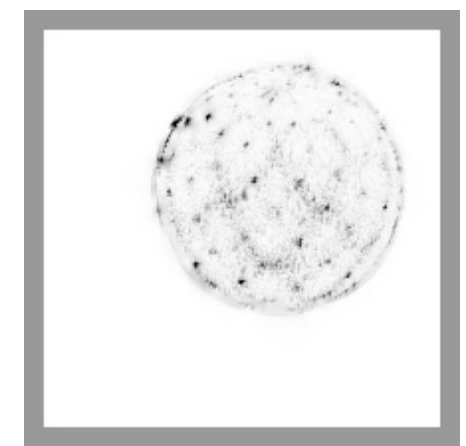

Figure 12.

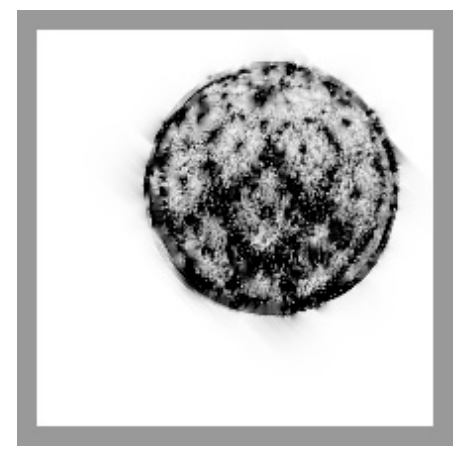

Figure 14.

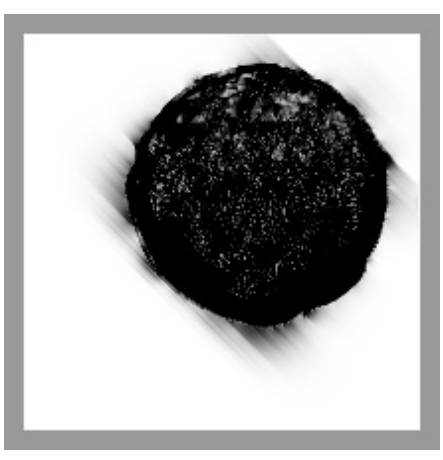

First series:

$q=2, R=0.125$

$A A E=4.15$

$M E E=11.04, I E E=11.12$
First series:

$q=2, R=4$

$A A E=4.11$,

$M E E=2.16, I E E=4.40$
First series:

$q=2, R=0.5$

$A A E=4.22$,

$M E E=7.25, I E E=7.89$

Figure 16. 


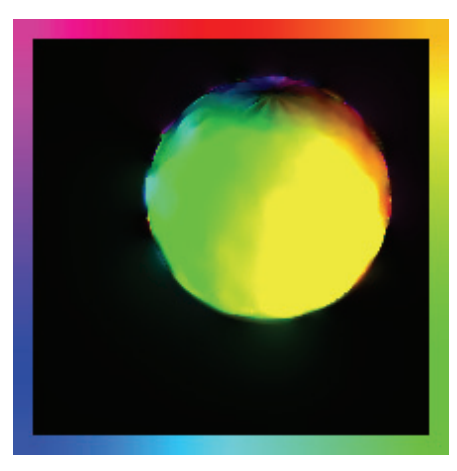

Figure 17.

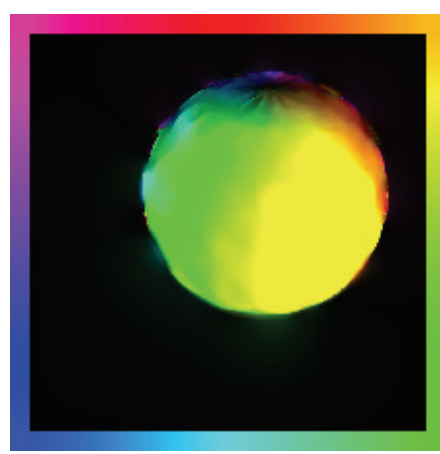

Figure 19.

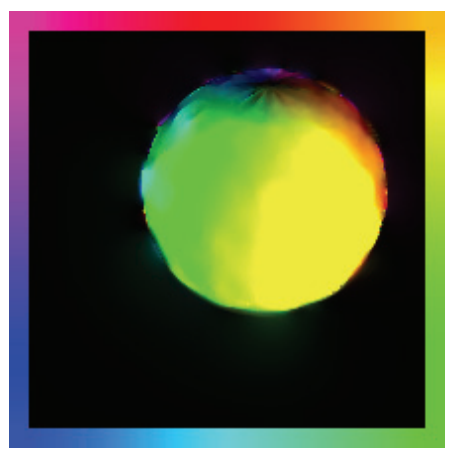

Figure 21 .

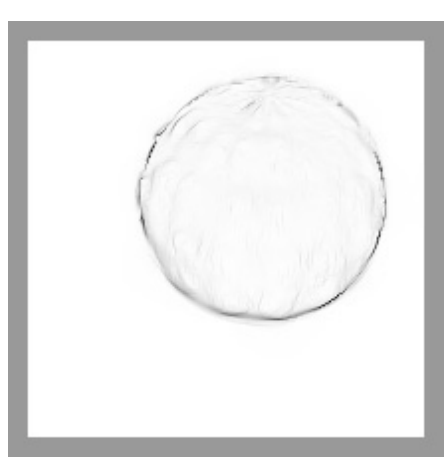

Figure 18.

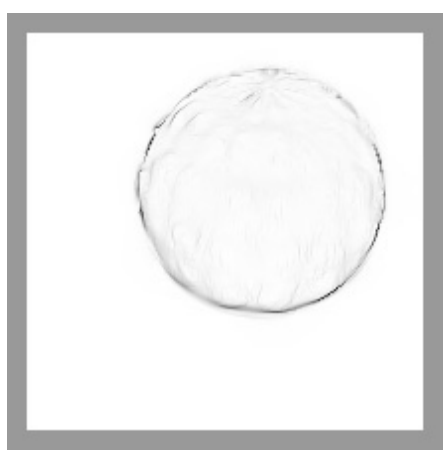

Figure 20.

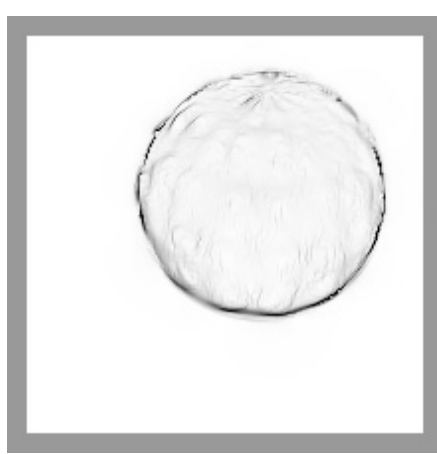

Second series:

$\varepsilon=0.001, \mu=0.001, p=2$, $q=2, R=1, \alpha=0$

$A A E=3.08$,

$M E E=2.19, I E E=4.37$
Second series:

$\varepsilon=0.001, \mu=0.001, p=2$,

$q=2, R=4, \alpha=0$

$A A E=3.08$,

$M E E=1.89, I E E=4.28$
Second series:

$\varepsilon=0.001, \mu=0.001, p=2$, $q=2, R=2, \alpha=0$

$A A E=3.07$,

$M E E=1.89, I E E=4.28$

Figure 22. 


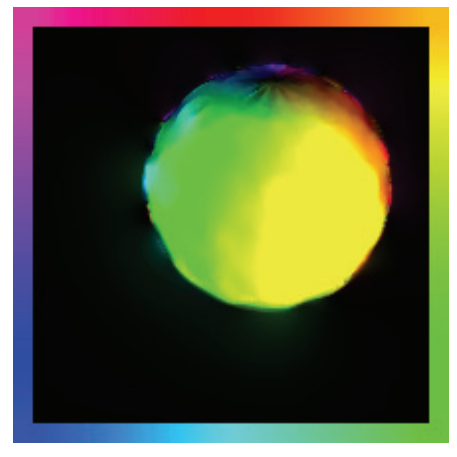

Figure 23.

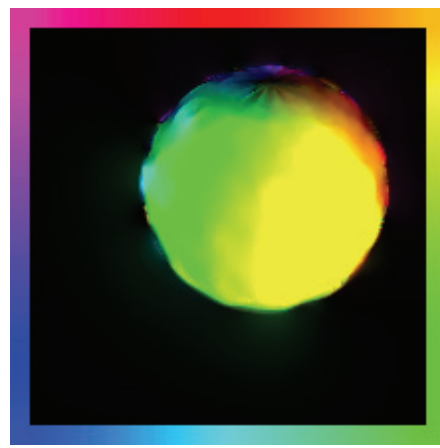

Figure 25.

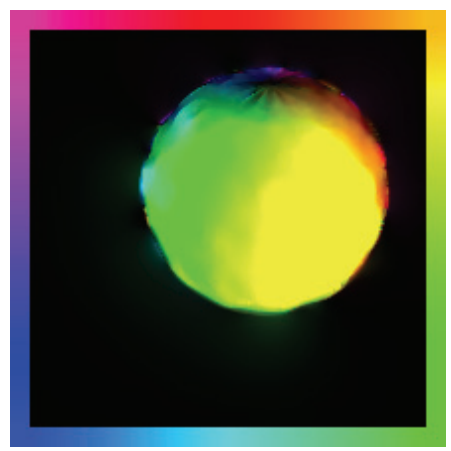

Figure 27.

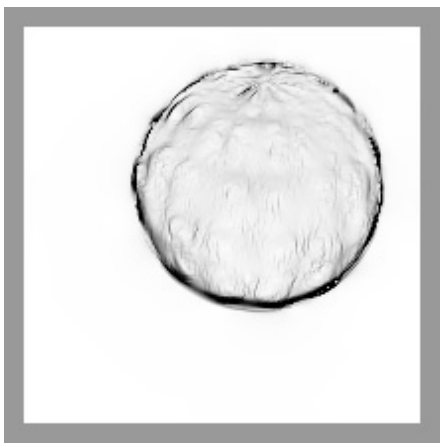

Figure 24 .

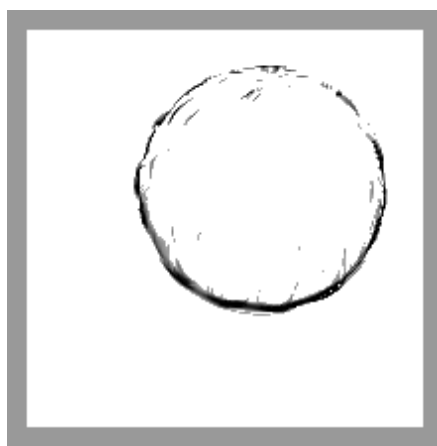

Figure 26 .

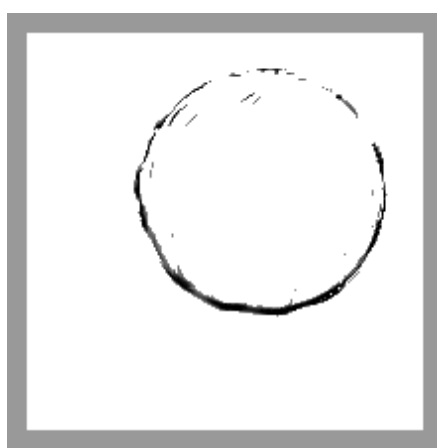

Second series:

$\varepsilon=0.001, \mu=0.001, p=2$, $q=2, R=0.5, \alpha=0.2$

$A A E=3.23$,

$M E E=2.82, I E E=4.78$
Second series:

$\varepsilon=0.001, \mu=0.001, p=2$,

$q=2, R=0.5, \alpha=0$

$A A E=3.23$,

$M E E=3.03, I E E=4.72$

Second series:

$\varepsilon=0.001, \mu=0.001, p=2$,

$q=2, R=0.5, \alpha=0.1$

$A A E=3.23$

$M E E=2.87, I E E=4.78$

Figure 28. 


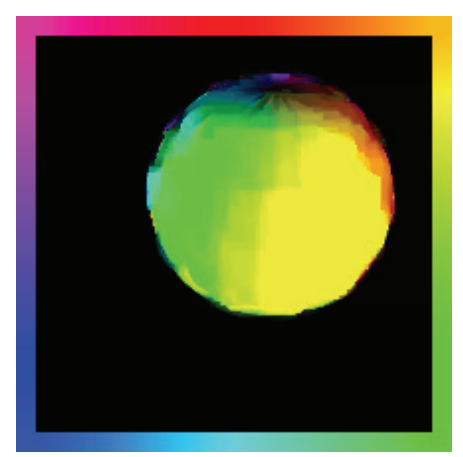

Figure 29.

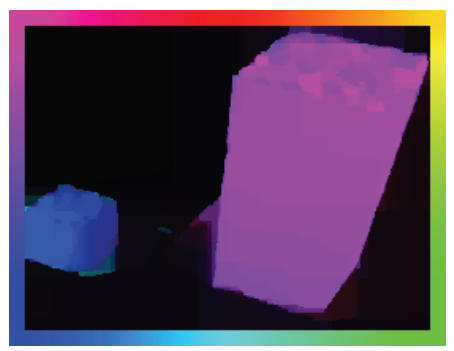

Figure 31 .

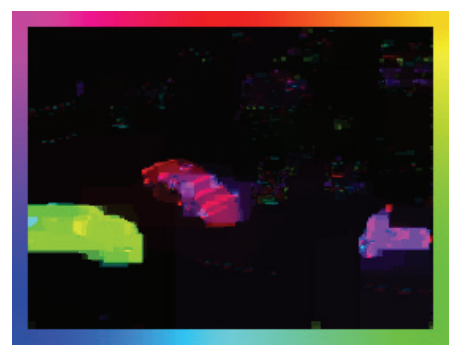

Figure 33.

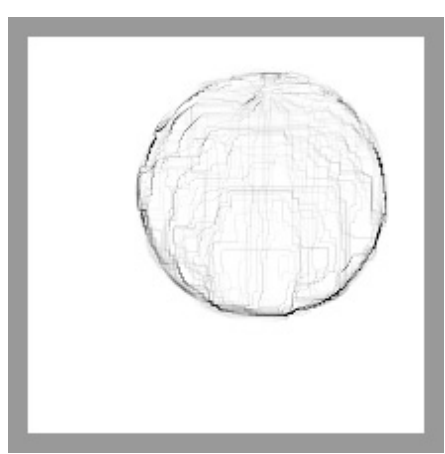

Figure 30 .

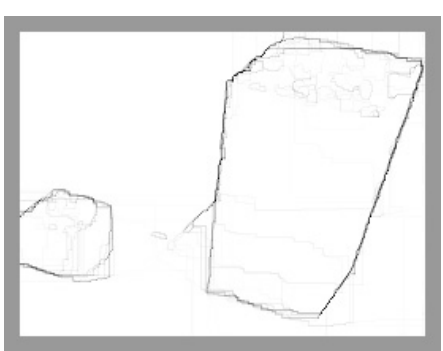

Figure 32.

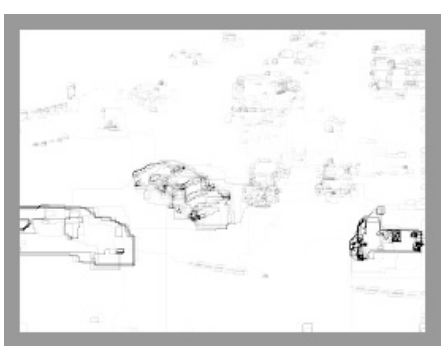

Third series:

$\varepsilon=0.001, \mu=0.01, R=2$

$A A E, M E E$, and $I E E$ could not be calculated since groundtruth data are not available.
Third series:

$\varepsilon=0.001, \mu=0.002, R=2$

$A A E=2.57$

$M E E=2.11, I E E=4.38$

Third series:

$\varepsilon=0.001, \mu=0.07, R=2$

$A A E=6.44$,

$M E E=2.87, I E E=8.84$

Figure 34. 


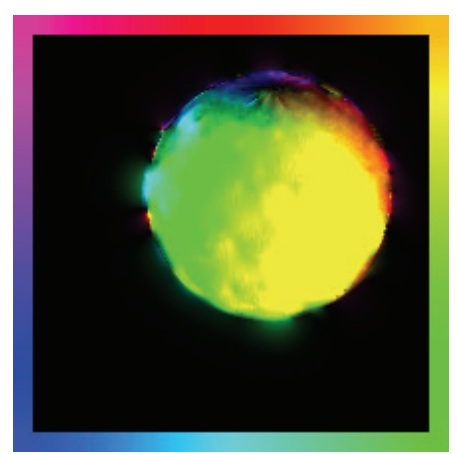

Figure 35.

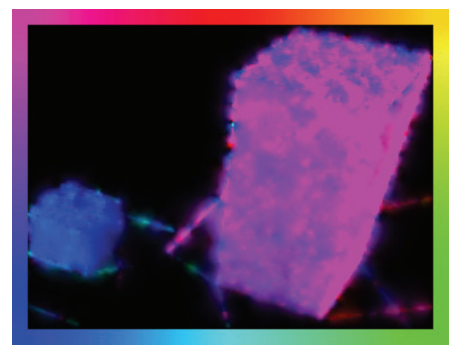

Figure 37.

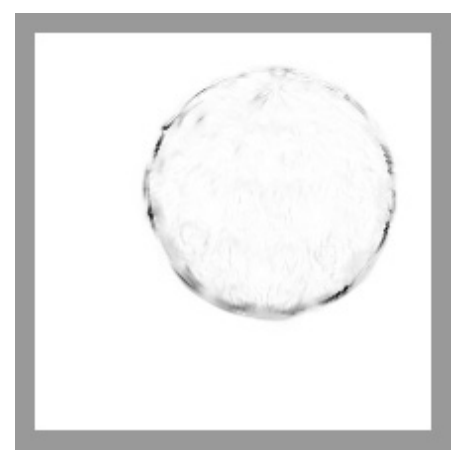

Figure 36 .

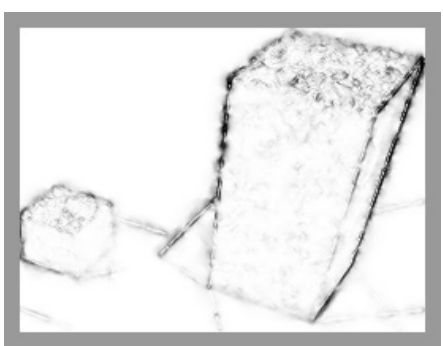

Ambrosio-Tortorelli method:

$\varepsilon=0.005, c_{1}(\varepsilon)=40$,

$c_{2}(\varepsilon)=1, c_{3}(\varepsilon)=0.5$,

$c_{4}(\varepsilon)=0.5$

$A A E=7.39$,

$M E E=3.61, I E E=8.75$ $\varepsilon=0.005, c_{1}(\varepsilon)=1250$,

$c_{2}(\varepsilon)=3, c_{3}(\varepsilon)=1, c_{4}(\varepsilon)=0$

$A A E=2.99$,

$M E E=1.89, I E E=4.30$

Figure 38.

\section{REFERENCES}

[1] L. Ambrosio And V. M. Tortorelli, Approximation of functionals depending on jumps by elliptic functionals via Г-convergence, Comm. Pure Appl. Math., 43 (1990), pp. 999-1036.

[2] L. Ambrosio And V. M. Tortorelli, On the approximation of free discontinuity problems, Boll. Un. Mat. Ital. B (7), 6 (1992), pp. 105-123.

[3] G. Aubert, R. Deriche, And P. Kornprobst, Computing optical flow via variational techniques, SIAM J. Appl. Math., 60 (1999), pp. 156-182.

[4] G. Aubert and P. Kornprobst, Mathematical Problems in Image Processing: Partial Differential Equations and the Calculus of Variations, 2nd ed., Springer, New York, 2006.

[5] G. Bellettini And A. Coscia, Discrete approximation of a free discontinuity problem, Numer. Funct. Anal. Optim., 15 (1994), pp. 201-224.

[6] B. Bourdin, Image segmentation with a finite element method, M2AN Math. Model. Numer. Anal., 33 (1999), pp. 229-244.

[7] T. Brox, A. Bruhn, And J. Weickert, Variational motion segmentation with level sets, in Computer Vision-ECCV 2006, Lecture Notes in Comput. Sci. 3951, H. Bischof, A. Leonardis, and A. Pinz, eds., Springer, Berlin, Heidelberg, 2006, pp. 471-483.

[8] A. Bruhn, J. Weickert, T. Kohlberger, and C. Schnörr, A multigrid platform for real-time motion computation with discontinuity-preserving variational methods, Int. J. Comput. Vision, 70 (2006), pp. $257-277$.

[9] A. Bruhn, J. Weickert, And C. Schnörr, Lucas/Kanade meets Horn/Schunck: Combining local and global optic flow methods, Int. J. Comput. Vision, 61 (2005), pp. 211-231.

[10] C. Brune, Berechnung des Optischen Flusses und Kantenerkennung mit Optimierungsmethoden, Diploma thesis, Institut für Numerische und Angewandte Mathematik, Westfälische Wilhelms-Universität Münster, Münster, Germany, 2007.

Copyright (C) by SIAM. Unauthorized reproduction of this article is prohibited. 
[11] A. Chambolle, An algorithm for total variation minimization and applications, J. Math. Imaging Vision, 20 (2004), pp. 89-97.

[12] B. Dacorogna, Direct Methods in the Calculus of Variations, 2nd ed., Springer, New York, 2008.

[13] G. Deweß And P. Helbig, Einschätzen und Optimieren von Verkehrsflüssen, in Mathematik in der Praxis. Fallstudien aus Industrie, Wirtschaft, Naturwissenschaften und Medizin, A. Bachem, M. Jünger, and R. Schrader, eds., Springer, Berlin, 1995, pp. 473-492.

[14] W. Enkelmann, Investigations of multigrid algorithms for the estimation of optical flow fields in image sequences, Comput. Vision Graph. Image Process., 43 (1988), pp. 150-177.

[15] L. C. Evans And R. F. Gariepy, Measure Theory and Fine Properties of Functions, CRC Press, Boca Raton, FL, 1992.

[16] R. Fourer, D. M. Gay, And B. W. Kernighan, AMPL: A Modeling Language for Mathematical Programming, 2nd ed., Brooks/Cole-Thomson Learning, Pacific Grove, CA, 2003.

[17] L. Franek, M. Franek, H. Maurer, and M. Wagner, Image Restoration and Simultaneous Edge Detection by Optimal Control Methods, Preprint-Reihe Mathematik, Preprint Nr. M-05/2008, BTU Cottbus, Cottbus, Germany; Optimal Control Appl. Methods, to appear.

[18] T. Gandhi, S. Devadiga, R. Kasturi, and O. Camps, Detection of obstacles on runways using egomotion compensation and tracking of significant features, Image Vision Comput., 18 (2000), pp. 805-815.

[19] H. Grossauer, Inpainting of movies using optical flow, in Mathematical Models for Registration and Applications to Medical Imaging, Math. Ind. 10, O. Scherzer, ed., Springer, Berlin, 2006, pp. 151-162.

[20] L. He, M. Burger, AND S. J. Osher, Iterative total variation regularization with non-quadratic fidelity, J. Math. Imaging Vision, 26 (2006), pp. 167-184.

[21] W. Hinterberger and O. Scherzer, Models for image interpolation based on the optical flow, Computing, 66 (2001), pp. 231-247.

[22] W. Hinterberger, O. Scherzer, C. Schnörr, And J. Weickert, Analysis of optical flow models in the framework of the calculus of variations, Numer. Funct. Anal. Optim., 23 (2002), pp. 69-89.

[23] J.-B. Hiriart-Urruty and C. Lemaréchal, Convex Analysis and Minimization Algorithms. I. Fundamentals, Grundlehren Math. Wiss. 305, Springer, Berlin, 1993.

[24] B. K. P. Horn And B. G. Schunck, Determining optical flow, Artificial Intelligence, 17 (1981), pp. 185-203.

[25] B. Jansen, Interior Point Techniques in Optimization, Kluwer Academic Publishers, Dordrecht, The Netherlands, 1997.

[26] B. Jung And G. S. Sukhatme, Real-Time Motion Tracking from a Mobile Robot, Technical Report CRES-05-008, Center for Robotics and Embedded Systems, University of Southern California, Los Angeles.

[27] P. Kornprobst, R. Deriche, And G. Aubert, Image sequence analysis via partial differential equations, J. Math. Imaging Vision, 11 (1999), pp. 5-26.

[28] A. Kumar, S. Haker, A. Stillman, C. W. Curry, D. P. Giddens, A. R. Tannenbaum, and A. YEZzI, Total-variational-based optical flow for cardiac-wall motion tracking, in Medical Imaging 2001: Image Processing, Proc. SPIE 4322, M. Sonka and K. M. Hanson, eds., SPIE-International Society for Optical Engineering, Bellingham, WA, 2001, pp. 1550-1553.

[29] C. LAIRD AND A. WÄChTER, Introduction to IPOPT: A Tutorial for Downloading, Installing, and Using IPOPT. Revision No. 1024, http://www.coin-or.org/Ipopt/documentation/ (last access: 29.02.2008).

[30] H. Maurer and H. D. Mittelmann, Optimization techniques for solving elliptic control problems with control and state constraints. I. Boundary control, Comput. Optim. Appl., 16 (2000), pp. 29-55.

[31] H. Maurer and H. D. Mittelmann, Optimization techniques for solving elliptic control problems with control and state constraints. II. Distributed control, Comput. Optim. Appl., 18 (2001), pp. 141-160.

[32] B. McCane, K. Novins, D. Crannitch, and B. Galvin, On benchmarking optical flow, Comput. Vis. Image Underst., 84 (2001), pp. 126-143.

[33] P. NEsi, Variational approach to optical flow estimation managing discontinuities, Image Vision Comput., 11 (1993), pp. 419-439.

[34] N. Papenberg, A. Bruhn, T. Brox, S. Didas, and J. Weickert, Highly accurate optic flow computation with theoretically justified warping, Int. J. Comput. Vision, 67 (2006), pp. 141-158.

Copyright ( $\odot$ by SIAM. Unauthorized reproduction of this article is prohibited. 
[35] S. Pickenhain And M. Wagner, Critical points in relaxed deposit problems, in Calculus of Variations and Optimal Control, Chapman \& Hall/CRC Res. Notes Math. 411, A. Ioffe, S. Reich, and I. Shafrir, eds., Chapman \& Hall/CRC Press, Boca Raton, FL, 2000, pp. 217-236.

[36] K. N. Plataniotis and A. N. Venetsanopoulos, Color Image Processing and Applications, Springer, Berlin, Heidelberg, New York, 2000.

[37] O. Scherzer, M. Grasmair, H. Grossauer, M. Haltmeier, and F. Lenzen, Variational Methods in Imaging, Springer, New York, 2009.

[38] S. P. Sethi And G. L. Thompson, Optimal Control Theory: Applications to Management Science and Economics, 2nd ed., Springer, New York, 2006.

[39] N. Slesareva, A. Bruhn, And J. Weickert, Optic flow goes stereo: A variational method for estimating discontinuity-preserving dense disparity maps, in Pattern Recognition. Proceedings of the 27th DAGM Symposium, Vienna, Austria, 2005, Lecture Notes in Comput. Sci. 3663, W. Kropatsch, R. Sablatnig, and A. Hanbury, eds., Springer, Berlin, Heidelberg, 2005, pp. 33-40.

[40] A. Talukder and L. Matthies, Real-time detection of moving objects for moving vehicles using dense stereo and optical flow, in Proceedings of the 2004 IEEE/RSJ Conference on Intelligent Robots and Systems (IROS), Sendai, Japan, 2004, Vol. IV, IEEE, Piscataway, NJ, 2004, pp. 3718-3725.

[41] A. Telea, T. Preusser, C. Garbe, M. Droske, and M. Rumpf, A variational approach to joint denoising, edge detection and motion estimation, in Pattern Recognition. Proceedings of the 28th DAGM Symposium, Berlin, Germany, 2006, Lecture Notes in Comput. Sci. 4174, K. Franke, K.-R. Müller, B. Nickolay, and R. Schäfer, eds., Springer, Berlin, Heidelberg, 2006, pp. 525-535.

[42] K. Theissen, Optimale Steuerprozesse unter partiellen Differentialgleichungs-Restriktionen mit linear eingehender Steuerfunktion, Ph.D. thesis, Westfälische Wilhelms-Universität Münster, Münster, Germany, 2006.

[43] G. Vossen And H. Maurer, On $L^{1}$-minimization in optimal control and applications to robotics, Optimal Control Appl. Methods, 27 (2006), pp. 301-321.

[44] A. WÄChter AND L. T. BIEgleR, On the implementation of an interior-point filter line-search algorithm for large-scale nonlinear programming, Math. Program. Ser. A, 106 (2006), pp. 25-57.

[45] M. Wagner, Pontryagin's maximum principle for multidimensional control problems in image processing, J. Optim. Theory Appl., 140 (2009), pp. 543-576.

[46] J. Weickert AND T. BRox, Diffusion and regularization of vector-and matrix-valued images, in Inverse Problems, Image Analysis, and Medical Imaging, Contemp. Math. 313, M. Zuhair Nashed and O. Scherzer, eds., AMS, Providence, RI, 2002, pp. 251-268.

[47] J. WeICKERT AND C. SChNÖRR, A theoretical framework for convex regularizers in PDE-based computation of image motion, Int. J. Comput. Vision, 45 (2001), pp. 245-264.

[48] J. WEICKERT AND C. SCHNÖRR, Variational optic flow computation with a spatio-temporal smoothness constraint, J. Math. Imaging Vision, 14 (2001), pp. 245-255.

Copyright ( $\odot$ by SIAM. Unauthorized reproduction of this article is prohibited. 